\title{
Heartbeat: increased use of NOACs in patients with atrial fibrillation reduces health care system costs by reducing stroke incidence
}

doi:10.1136/heartjnl-2020-318709

A major goal in management of adults with atrial fibrillation (AF) is prevention of stroke. In an editorial, our stroke neurology colleagues ${ }^{1}$ point out that about $1 / 3$ of patients with an AF-related stroke had a diagnosis of AF before the stroke but were not on anticoagulation therapy. When vitamin K-antagonists were the only option for anticoagulation, 'many patients did not receive anticoagulant therapy despite a clear-cut indication due to a highly inconvenient treatment (repeated international normalised Ratio measurements, many food and drug-interactions, frequent dose adjustments) and a significant risk of intracerebral (and other major) bleeding.' Now, with the availability of non-vitamin $\mathrm{K}$ oral anticoagulants (NOACs) the risk-benefit balance has shifted towards therapy to prevent AF-related stroke. Still, hesitation remains due to the higher cost of these newer medications.

In order to better understand temporal changes in AF-related stroke incidence, anticoagulant medication prescribing and overall and per-patient costs in the UK, Orlowski and colleagues ${ }^{2}$ compared the time periods of 2011-2014 versus 20142017, using National Health Service data. A dramatic increase (over 85\%) in oral anticoagulation prescribing was seen, mostly due to increased use of NOACs (figure 1). As expected, greater use of NOACs was associated with an increase in total medication costs by over $780 \%$, mainly due to the increased number of AF patients being treated, corresponding to an increase in medication cost of about $51 \%$ per patient. However, the increased cost of medication was offset by a decrease in AF-related stroke incidence by $11 \%$, resulting in an overall incremental cost saving per patient of $£ 289$.

As Seiffge and Meinel $^{1}$ comment: 'Apparently, providing a convenient, safe anticoagulation therapy to a large number of patients is beneficial for everybody. Patients are protected from ischaemic

Correspondence to Professor Catherine M Otto, Division of Cardiology, University of Washington, Seattle,WA 98195, USA; cmotto@uw.edu

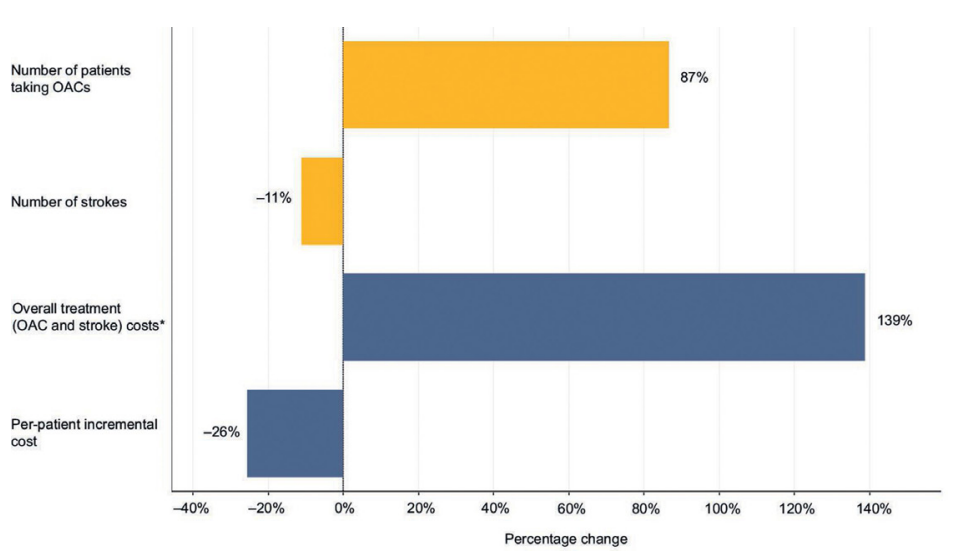

Figure 1 Overall percentage changes in numbers of patients and strokes and in total and incremental per-patient treatment costs between 2011-2014 and 2014-2017. *Calculated as the total prescribing costs for direct oral anticoagulants, warfarin and international normalised ratio monitoring plus management in the first year after stroke. OAC, oral anticoagulation.

stroke, the number of devastating intracerebral haemorrhages related to anticoagulant use does not increase and-due to the savings related to fewer strokes-there

is an overall saving for the healthcare system.'

The importance of continued cardiovascular monitoring for heart failure in

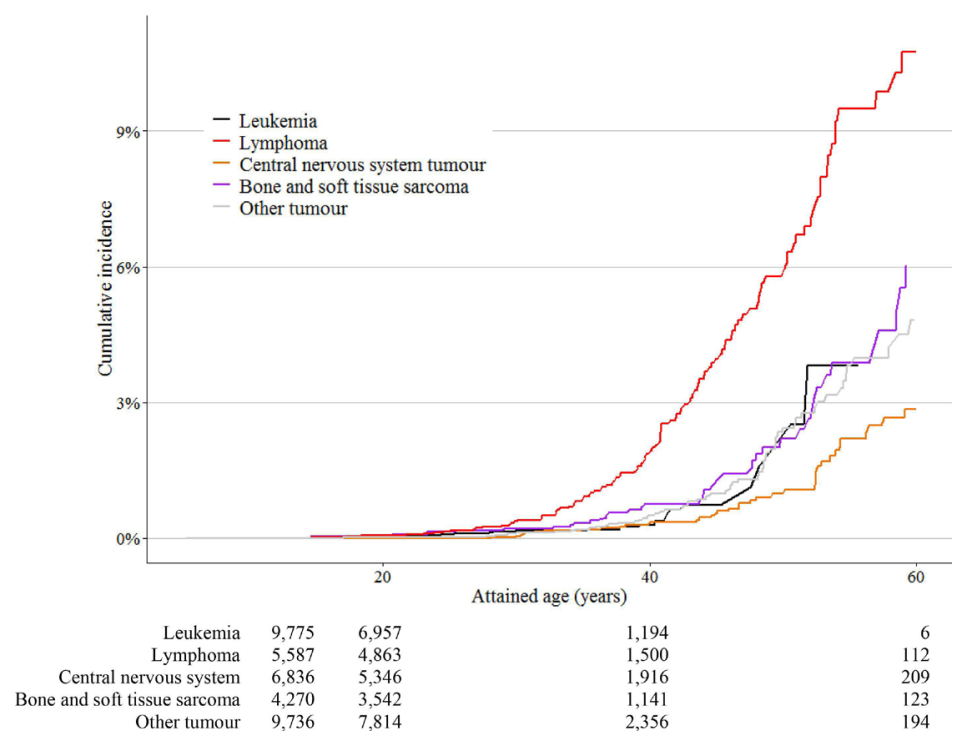

Figure 2 Cumulative incidence of symptomatic cardiac ischaemia per malignancy group with attained age as time scale. Unadjusted Gray's test: leukaemia versus lymphoma $p<0.0001$; leukaemia versus central nervous system (CNS) tumour $p=0.16$; leukaemia versus bone and soft tissue sarcoma $p=0.16$; leukaemia versus other tumour $p=0.72$; lymphoma versus $C N S$, bone and soft tissue sarcoma, other tumour $p<0.0001$; CNS versus bone and soft tissue sarcoma, other tumour $\mathrm{p}<0.0001$; bone and soft tissue sarcoma versus other tumour $\mathrm{p}=0.32$. 


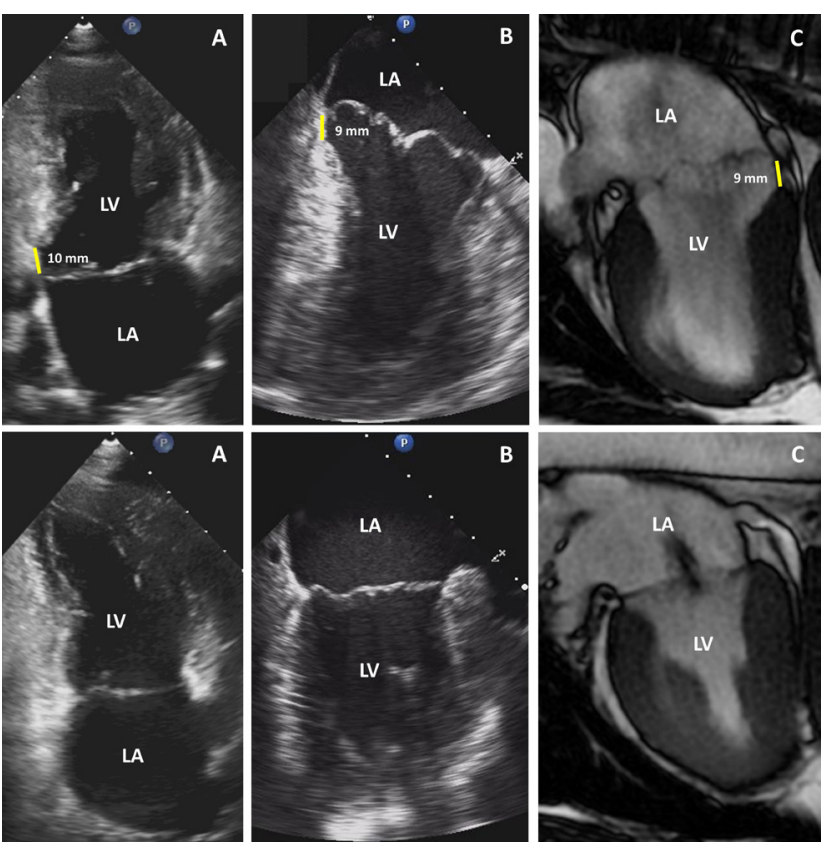

Figure 3 MAD evaluation by multimodality imaging. Shown are two examples of Barlow's disease, where the LA-posterior mitral valve annulus junction was assessed by transthoracic echocardiography (A), transoesophageal echocardiography (B) and cardiac magnetic resonance (C). The three techniques are concordant on presence (upper panels) and absence (lower panels) of MAD in two-chamber long-axis view, at P3 level. In the upper panels, MAD is identified (yellow line) and measured at end-systole. LA, left atrium; LV, left ventricle; MAD, mitral annular disjunction.

childhood cancer survivors (CCS) treated with potentially cardio-toxic medications is well known. The risk of symptomatic cardiac ischaemia has received less attention. In this issue of Heart, Feijen and colleagues $^{3}$ report a cumulative incidence of symptomatic cardiac ischaemia in CCS patients by age 60 of 5.4\% (95\% CI 4.6\% to $6.2 \%$ ) based on combined data from over 36200 patients. The risk of cardiac ischaemia was higher in men than women, in those who received chemotherapy and/

or radiation therapy compare to those treated with surgery alone, and was especially high in those with lymphoma (figure 2).

The strengths and limitations of this study are discussed in an editorial by Kok. ${ }^{4}$ He points out that : ' $15 \%$ of the events in the current study already occurred in those $<30$ years old and that the cumulative incidence risk curves in these studies indicate that CCS have similar incidences of severe cardiac ischaemic events as patients

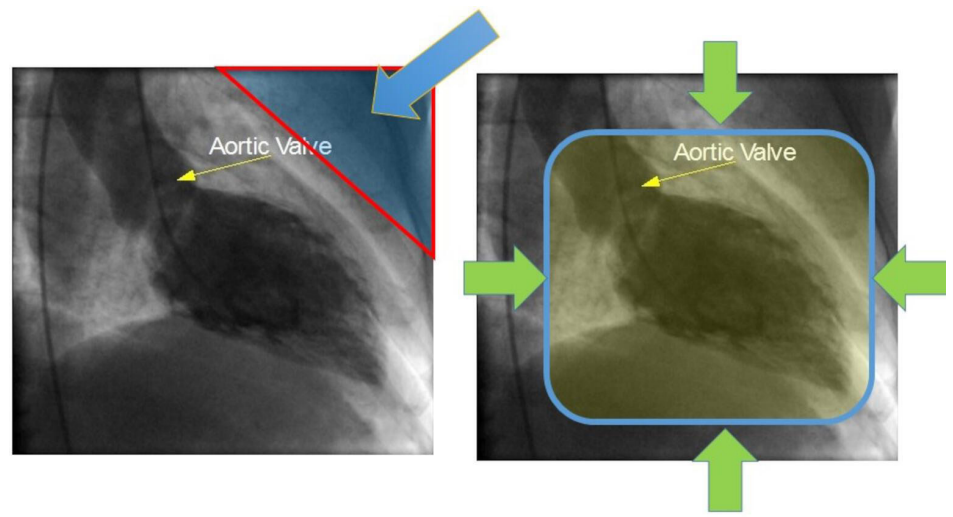

Figure 4 Collimators and shutters. Actively collimating to the volume of interest (green arrows) reduces the overall integral dose to the patient and thus minimises the radiation risk. Less volume irradiated will result in less X-ray scatter incident on the detector. This results in improved subject contrast and image quality. Applying shutters (blue arrow) allows a more uniform image and thus reduction in radiation.

in the general population who are $10-15$ years older. " Thus, 'The study of risk for the specific outcome of cardiac ischaemic events in models would open the possibility to prevent cardiovascular events with lifestyle interventions at an early age (hypertension as the most prominent risk factor) and also to better identify the CCS at low risk for this type of outcome.'

Another interesting study ${ }^{5}$ in this issue proposes more specific imaging approaches for mitral annular disjunction (MAD), a structural abnormality seen in some patients with mitral valve prolapse (MVP) that is associated with malignant arrhythmias (figure 3).

In an editorial, Haugga ${ }^{6}$ comments that 'Although there is debate whether MAD is an actual anatomical and clinical entity, the clinical interest in this anatomical abnormality has been revitalised recently linking MAD with ventricular arrhythmias and sudden cardiac death.' In the current study, 'Prevalence of concomitant MAD in patients with MVP increased according to imaging modality from $17 \%, 25 \%$ to $42 \%$ by transthoracic echocardiography, transoesophageal echocardiography and cardiac MRI, respectively.' For the future, 'outcome studies on how MAD affects surgical and transcatheter interventions are needed. Furthermore, we need strategies of management in incidental findings of MAD in an asymptomatic patient.'

The Education in Heart article ${ }^{7}$ in this issue provides a primer on risks of radiation exposure in the catheterisation laboratory and the methods to minimise exposure of patients and staff (figure 4). Essential reading for all who work in the catheterisation laboratory.

Clinical cardiologists will also want to look at the review article on treatment of premature ventricular contractions in patients with heart failure with reduced ejection fraction. ${ }^{8}$ Cardiac imagers will find the review article by Lindner ${ }^{9}{ }^{9}$ on contrast echocardiography interesting with a roadmap for future research for diagnosis and therapy using this technique. The Cardiology in Focus article, ${ }^{10}$

10 discuses the role of simulation training in cardiology, an especial topical issue given the constraints on conventional training with the COVID-19 pandemic.

Contributors Heartbeat for Volume 107 Issue 1.

Funding The authors have not declared a specific grant for this research from any funding agency in the public, commercial or not-for-profit sectors.

Competing interests None declared. 
Patient and public involvement Patients and/ or the public were not involved in the design, or conduct, or reporting, or dissemination plans of this research.

Patient consent for publication Not required.

Provenance and peer review Commissioned; internally peer reviewed.

(c) Author(s) (or their employer(s)) 2021. No commercial re-use. See rights and permissions. Published by BMJ.

\section{D) Check for updates}

To cite Otto CM. Heart 2021;107:1-3.

Heart 2021;107:1-3.

doi:10.1136/heartjnl-2020-318709
ORCID iD

Catherine M Otto http://orcid.org/0000-0002-05279392

\section{REFERENCES}

1 Seiffge DJ, Meinel TR. The real prize of direct oral anticoagulant blockbuster. Heart 2021;107:8-9.

2 Orlowski A, Gale CP, Ashton R, et al. Clinical and budget impacts of changes in oral anticoagulation prescribing for atrial fibrillation. Heart 2021;107:47-53.

3 Feijen EAM, van Dalen EC, van der Pal HJH, et al. Increased risk of cardiac ischaemia in a pan-European cohort of 36205 childhood cancer survivors: a PanCareSurFup study. Heart 2021;107:33-40.

4 Kok WE. Too often and too early: the risk of cardiac ischaemia in adult survivors of childhood cancer. Heart 2021;107:6-7.
5 Mantegazza V, Volpato V, Gripari P, et al. Multimodality imaging assessment of mitral annular disjunction in mitral valve prolapse. Heart 2021;107:25-32.

6 Haugaa K. Improving the imaging diagnosis of mitral annular disjunction. Heart 2021;107:4-5.

7 Roguin A, Nolan J. Radiation protection in the cardiac catheterisation lab: best practice. Heart 2021;107:76-82.

8 Mulder BA, Rienstra M, Blaauw Y. Evaluation and treatment of premature ventricular contractions in heart failure with reduced ejection fraction. Heart 2021;107:10-7.

9 Lindner JR. Contrast echocardiography: current status and future directions. Heart 2021;107:18-24.

10 Moss A, Stoll VM. Simulation training for the cardiology trainee. Heart 2021;107:83-4. 